\title{
INFLUÊNCIA DE SUBSTRATOS E FUNGOS MICORRÍZICOS ARBUSCULARES NO DESENVOLVIMENTO VEGETATIVO DO PORTA-ENXERTO FLYING DRAGON (Poncirus trifoliata, var. montruosa Swing.) ${ }^{1}$
}

\author{
PAULO VITOR DUTRADE SOUZA², EDGAR CARNIEL ${ }^{3}$, JOSÉANTÔNIO KRÖEFF SCHMITZ4 ${ }^{4}$ SAMAR VELHO DA SILVEIRA $^{5}$
}

\begin{abstract}
RESUMO - O presente estudo teve por objetivos avaliar o efeito da composição do substrato e da inoculação de duas espécies de fungos micorrízicos arbusculares (FMA) (Glomus clarum e Acaulospora scrobiculata) sobre o desenvolvimento vegetativo, o conteúdo em substâncias de reserva nos tecidos e a percentagem de colonização radicular por FMA no porta-enxerto Flying dragon (Poncirus trifoliata, var. monstruosa Swing). Os tratamentos consistiram de dois substratos: $\mathrm{S} 1=$ solo + areia (1:1; v:v) e S2 = solo + areia + resíduo decomposto de casca de acácia-negra (2:2:1; v:v:v), ambos com e sem inoculação isolada de duas espécies de FMA (G. clarum e A. scrobiculata). Constatou-se que a adição de resíduo decomposto de casca de acácia-negra melhorou as características químicas e físicas do substrato, permitindo um maior desenvolvimento vegetativo e acúmulo de substâncias de reserva às plantas de Flying dragon em relação ao substrato solo + areia. A eficiência da simbiose foi variável com o substrato e com a espécie de FMA, onde o efeito positivo dos FMA foi notado apenas no substrato solo + areia, mais pobre nutricionalmente, sendo A. scrobiculata a espécie mais eficiente.
\end{abstract}

Termos para Indexação: Citricultura, endomicorrizas; porta-enxertos; propagação.

\section{INFLUENCE OF GROWING MEDIA AND ARBUSCULAR MYCORRHIZAL FUNGI IN THE VEGETATIVE DEVELOPMENT OF FLYING DRAGON (Poncirus trifoliata, var. monstruosa Swing.) ROOTSTOCK}

\begin{abstract}
The present study had the aim of evaluating the effects of substrate composition and the inoculation of two species of arbuscular mycorrhizal fungi (AMF) (Glomus clarum and Acaulospora scrobiculata) on the vegetative development, carbohydrate contents and percentage of radicular colonization settling by AMF in Flying Dragon (Poncirus trifoliata, var. monstruosa Swing.) rootstock. The treatments consisted of two substrates: $\mathrm{S} 1=$ soil + sand (1:1; v: v), and S2 = soil + sand + decomposed residue of acacia, (2:2:1; v: v: v). Both treatments were submitted to inoculation with two species of AMF (G. clarum e A. scrobiculata) and no inoculation. The addition of decomposed residue of acacia bark in S2, improved the chemical and physical properties of the substrate, resulting in a better vegetative development of Flying Dragon plants. Also a significant interaction between type of substrate and effect of AMF on dry matter (aerial part and roots) and carbohydrate contents was determined. The efficiency of symbiosis varied according to substrate composition and AMF species. A positive effect of AMF was noted when the mixture soil + sand was used, a poor substrate, being A. scrobiculate the most efficient species.
\end{abstract}

Index Terms: Citriculture, endomycorrhizae, rootstocks, propagation.

\section{INTRODUÇÃO}

A citricultura brasileira está enxertada sobre um número reduzido de porta-enxertos, tornando-a vulnerável às novas enfermidades. Os pomares do centro do País estão enxertados, principalmente, sobre limoeiro-cravo, enquanto, no Rio Grande do Sul, aproximadamente $90 \%$ da citricultura está enxertada sobre o Poncirus trifoliata (Schäfer \& Dornelles, 2000). Portanto, faz-se necessário estudos sobre o comportamento de diferentes porta-enxertos, visando a sua diversificação na citricultura (Schäfer, 2004; Fochesato, 2005).

Além disso, a fruticultura moderna objetiva reduzir custos através do cultivo de plantas com porte menor. O uso de porta-enxertos menos vigorosos, como o Flying Dragon (Poncirus trifoliata, var. monstruosa Swing.) propicia a produção de mudas com altura menor do que aquelas enxertadas em limão Cravo (Citrus limonia Osbeck) (Koller, 1994).

A produção de mudas cítricas em viveiros desprotegidos e diretamente no solo favorece a infestação por patógenos, como fungos do gênero Phytophthora (Programa Estadual de Citricultura, 1989). No Rio Grande do Sul, outra desvantagem do sistema tradicional é o longo período necessário para produção de mudas de citros ( 3 anos). O cultivo protegido, com uso de substrato livre de patógenos, permite em menos tempo a produção de mudas sadias (Schäfer, 2004; Fochesato, 2005).

A correta escolha do substrato é fundamental para a produção de mudas de qualidade, existindo materiais orgânicos com grande potencial para uso em citricultura (Schmitz et al., 2002). No Rio Grande do Sul, por exemplo, existem indústrias extratoras de tanino de Acácia-negra (Acacia meamsii De Wild.), gerando resíduos que podem poluir o meio ambiente. O uso desses resíduos como componentes de substrato propicia a obtenção de produtos alternativos eficientes, mais baratos e que permitem preservar o meio ambiente. $\mathrm{O}$ seu emprego, em misturas com outros materiais, como solo e areia, pode melhorar as características físicas e químicas do substrato e reduzir o uso de solo nas misturas.

Por sua vez, em ambiente protegido, recomenda-se o uso de substratos inertes ou desinfestados para evitar a contaminação por patógenos. Porém, também são eliminados os fungos micorrízicos arbusculares (FMA), os quais são fungos benéficos que incrementam o crescimento das mudas cítricas e reduzem os estresse do transplante. Nesse sistema de produção, o emprego de FMA torna-se importante, porém o mutualismo planta-FMA é influenciado pela cultivar de citros, pela espécie de FMA em questão e pelas características do substrato empregado (Ishii \& Kadoya, 1994).

O presente estudo objetivou testar o efeito do resíduo decomposto de casca de acácia-negra adicionada a um substrato solo + areia, o efeito de FMA e a interação destes fatores sobre o desenvolvimento vegetativo, conteúdo em substâncias de reserva nos tecidos e percentagem de colonização radicular por FMA em porta-enxertos de Flying Dragon.

\section{MATERIALEMÉTODOS}

O experimento foi conduzido em casa de vegetação no Setor de Horticultura da Estação Experimental Agronômica da Universidade Federal do Rio Grande do Sul, km 146, BR 290, município de Eldorado do Sul-RS. A variedade porta-enxerto utilizada foi o trifoliata Flying Dragon

\footnotetext{
(Trabalho 175/2004). Recebido: 31/08/2005. Aceito para publicação: 07/07/2005. Estudo Financiado pela Finep.

${ }^{2}$ Eng. Agr., Dr., Dep. Hortic. e Silvic., Fac. Agronomia, UFRGS, Av. Bento Gonçalves, 7712, C. Postal 15.100, Porto Alegre-RS. Bolsista CNPq.E-mail: pvdsouza@ufrgs.br.

${ }^{3}$ Eng. Agr., M. Sc., Fac. Agronomia, UFRGS. Av. Bento Gonçalves, 7712, C. P. 15.100, Porto Alegre-RS.

${ }^{4}$ Eng. Agr., Dr., FEPAGRO, Av. Gonçalves Dias, n. 570, CEP. 90.310-060, Porto Alegre-RS. Email: jose-schmitz@fepagro.rs.gov.br.

${ }^{5}$ Eng. Agr., M.Sc., Doutorando em Fitotecnia, Fac. de Agronomia, UFRGS, Bolsista CNPq. Email: svelho@eez.csic.es.
} 
(P. trifoliata) cultivada em dois substratos (S1: solo + areia, 1:1, v:v; S2: solo + areia + resíduo decomposto de casca de acácia-negra, 2:2:1, v:v:v). Testaram-se três tratamentos, utilizando FMA (Glomus clarum Nicol. \& Schenck, Acaulospora scrobiculata Trappe e uma testemunha nãoinoculada). O solo foi coletado do Horizonte B, Argissolo Vermelho distrófico típico, unidade de mapeamento São Gerônimo (Embrapa, 1999) na Estação Experimental Agronômica da UFRGS. A areia utilizada possuía granulometria média (entre 0,6 e $1 \mathrm{~mm}$ ). O resíduo decomposto de casca de Acácia-negra foi coletado em um depósito da empresa TANAC/SA (Montenegro-RS). Os substratos sofreram desinfestação prévia com solução de formolaldeído a $7 \%$.

Foram analisadas para os dois substratos as seguintes características químicas: pH em água, teor total de sais solúveis (TTSS), capacidade de troca de cátions (CTC) e teor de carbono orgânico. As características físicas analisadas foram: densidade seca, porosidade total, espaço de aeração, água disponível, água facilmente disponível, água tamponante e água remanescente. Essas análises foram realizadas no Laboratório de Biotecnologia do Departamento de Horticultura e Silvicultura da Faculdade de Agronomia da UFRGS. Para a determinação da porosidade total, espaço de aeração e água disponível, os materiais componentes foram caracterizados seguindo a metodologia proposta por De Boodt \& Verdonck (1972).

A semeadura foi feita em bandejas alveoladas de isopor (156 $\mathrm{ml} /$ alvéolo), colocando-se duas sementes por alvéolo. Após a germinação, selecionou-se uma plântula por alvéolo. Os tratamentos micorrizados receberam $10 \mathrm{~g} /$ alvéolo de solo rizosférico e raízes de aveia (Avena strigosa) colonizadas com as espécies de FMA. A adição do inóculo foi feita imediatamente antes da semeadura. A testemunha não recebeu inóculo de FMA.

Quatro meses após a semeadura, avaliaram-se a altura (desde o colo até o ápice) e o diâmetro do colo das plantas. Avaliou-se o peso seco das mesmas, após mantê-las em estufa, a $65^{\circ} \mathrm{C}$, até a obtenção do peso constante. Determinou-se o teor de substâncias de reserva nos tecidos, segundo método descrito por Priestley, citado por Souza (1990).

Para a determinação da colonização radicular com FMA, utilizouse metodologia descrita por Phillips \& Hayman, citada por Souza (1990).

O delineamento experimental utilizado foi o de blocos casualizados, com 10 plantas por parcela e quatro repetições. Para a interpretação dos resultados, foi utilizada a análise de variância, sendo a significância das diferenças entre as médias avaliada pelo teste de Duncan, a $5 \%$ de probabilidade.

\section{RESULTADOS E DISCUSSÃO}

A adição de uma fonte de matéria orgânica tornou o substrato 2 (S2) mais ácido, adicionou sais, cátions e carbono orgânico em relação ao substrato 1 (S1) (Tabela 1). Comparando-se os valores obtidos neste experimento com dados da literatura (Verdonck et al., 1981; Penningsfeld, 1983; Kämpf, 2000), observou-se que enquanto o S 1 apresentou valores de $\mathrm{pH}$ e de TTSS mais adequados, o S2 apresentou melhores índices de CTC e carbono orgânico.

Considerando-se que os componentes orgânicos, formadores de agregados estruturais, são as únicas fontes de nitrogênio no substrato, excetuando-se a adubação química, e fonte de outros nutrientes para as plantas (principalmente enxofre e fósforo), conclui-se que S2 é um substrato mais rico em nutrientes do que $\mathrm{S} 1$, apresentando um teor de salinidade um pouco mais elevado, mas não em níveis tóxicos, mesmo para espécies cítricas, consideradas plantas sensíveis à salinidade, segundo Black, citado por Oliveira (1991).

Em relação às características físicas, observa-se que a adição de um material orgânico (RDCA) em S2 melhorou consideravelmente a densidade seca, a porosidade total, o espaço de aeração, a água disponível, a água facilmente disponível, a água tamponante e a água remanescente, em relação a S1, comparando-se com valores de literatura (De Boodt \& Verdonck,1972; Penningsfeld, 1983).

A densidade seca mais elevada de $\mathrm{S} 1$, em relação a $\mathrm{S} 2$, diminuiu a porosidade total, gerando menor espaço de aeração e menor
TABELA 1 - Características químicas e físicas de dois substratos, antes de serem cultivados com plantas de Flying Dragon (Poncirus trifoliata, var. monstruosa Swing.). Porto Alegre, 1997.

\begin{tabular}{lcc}
\hline Características Químicas & $\mathrm{S} 1$ & $\mathrm{~S} 2$ \\
\hline $\mathrm{pH}$ & 5,8 & 5,4 \\
TTSS $\left(\mathrm{g} \mathrm{L}^{-1}\right)$ Teor Total de Sais Solúveis & 0,35 & 0,83 \\
CTC $\left(\mathrm{cmol}_{\mathrm{c}} \mathrm{L}^{-1}\right)$ Capacidade de Troca de Cátions & 3,8 & 15,5 \\
Carbono Orgânico $\left(\mathrm{C}_{\text {org }}, \% \mathrm{MS}\right)$ & 0,8 & 3,7 \\
Características Físicas & & \\
DS $\left(\mathrm{kg} \mathrm{m}^{-3}\right)$ & $1.487,0$ & $1.062,0$ \\
PT $\left(\mathrm{m}^{3} \mathrm{~m}^{-3}\right)$ & 0,406 & 0,602 \\
EA $\left(\mathrm{m}^{3} \mathrm{~m}^{-3}\right)$ & 0,181 & 0,268 \\
AD $\left(\mathrm{m}^{3} \mathrm{~m}^{-3}\right)$ & 0,089 & 0,158 \\
AFD $\left(\mathrm{m}^{3} \mathrm{~m}^{-3}\right)$ & 0,077 & 0,156 \\
AT $\left(\mathrm{m}^{3} \mathrm{~m}^{-3}\right)$ & 0,12 & 0,002 \\
AR-100 $\left(\mathrm{m}^{3} \mathrm{~m}^{-3}\right)$ & 0,136 & 0,176 \\
\hline
\end{tabular}

S1: solo + areia, 1:1, v:v; S2: solo + areia + resíduo decomposto de casca de acácianegra, 2:2:1, v:v:v; Carbono Orgânico ( $\left.\mathrm{C}_{\text {org }}\right)$; Densidade Seca (DS); Porosidade Total (PT); Espaço de Aeração (EA); Água Disponível (AD); Água Facilmente Disponível (AFD); Água Tamponante (AT); Água Remanescente (AR-100);

disponibilidade de água às plantas (Tabela 1). Observou-se, no S2, que o volume de água disponível e de água facilmente disponível quase duplicou em relação a S1 (Tabela 1), facilitando, portanto, a absorção de água pelas plantas nele cultivadas.

A melhor composição física e química do S2, em relação ao S1, redundou em maior altura das plantas em Flying Dragon (Tabela 2). Os FMAs testados não influíram significativamente sobre este parâmetro de avaliação. O diâmetro do colo das plantas não foi afetado pelos tratamentos testados (Tabela 2).

Encontrou-se uma interação significativa entre efeito de substrato e de espécies de FMA para matéria seca da parte aérea e raízes (Tabela 2). Verificou-se que a inoculação com FMA trouxe benefícios ao desenvolvimento vegetativo do Flying Dragon, quando as plantas foram cultivadas no $\mathrm{S} 1$, onde $A$. scrobiculata proporcionou maior matéria seca da parte aérea e raízes (Tabela 2). Ao contrário, quando cultivadas no S2, as plantas de Flying Dragon foram prejudicadas pelo FMA, principalmente pela presença de $A$. scrobiculata.

À semelhança do ocorrido para matéria seca, em S1 o conteúdo em substâncias de reserva nos tecidos das plantas micorrizadas foi superior às não-micorrizadas, não se observando o mesmo em S2 (Figura 1).

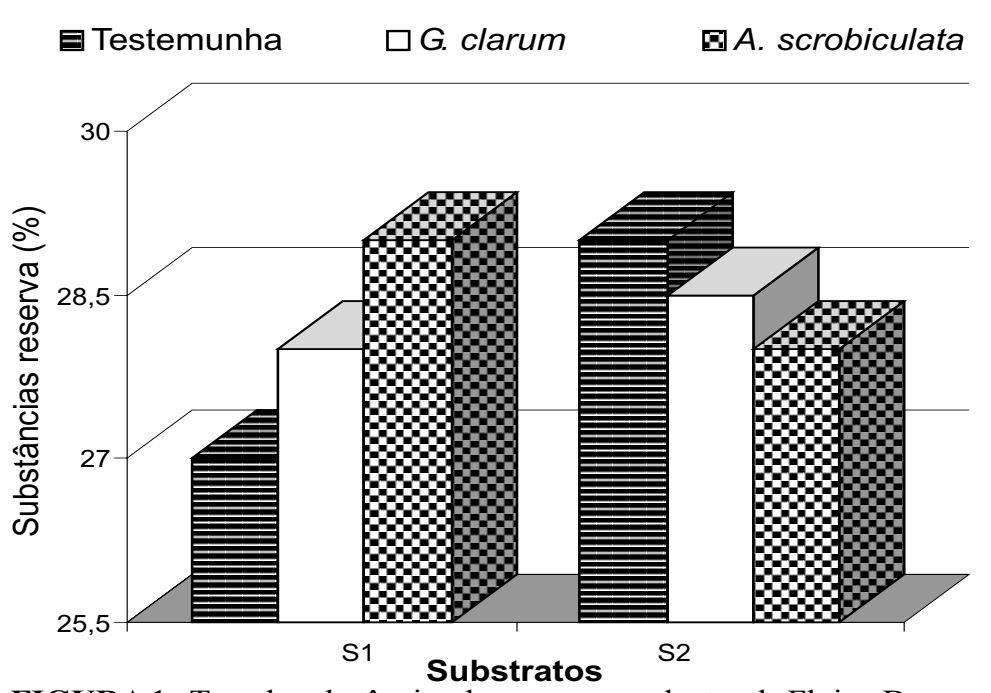

FIGURA 1 - Teor de substâncias de reserva em plantas de Flying Dragon (Poncirus trifoliata, var. monstruosa Swing.) cultivadas em 2 substratos (S1: solo + areia, v:v; S2: solo + areia+ resíduo decomposto de casca de acácia-negra, v:v:v) e inoculadas com FMA (G. clarum e A. scrobiculata). Barras seguidas de mesma letra não diferem entre si, ao nível de $5 \%$, pelo teste de Duncan. 
TABELA 2 - Altura, diâmetro do colo, matéria seca da parte aérea e das raízes de plantas de Flying Dragon (Poncirus trifoliata, var. monstruosa Swing.), cultivadas em dois substratos e inoculadas com fungos micorrízicos arbusculares. Porto Alegre, 1997.

\begin{tabular}{|c|c|c|c|c|c|c|}
\hline & \multicolumn{3}{|c|}{ Altura (mm) } & \multicolumn{3}{|c|}{ Diâmetro do colo (mm) } \\
\hline & Testemunha & G. clarum & A. Scrobiculata & Testemunha & G. clarum & A. Scrobiculata \\
\hline S1 & 58,50 & 62,50 & 60,75 & 1,63 & 1,68 & 1,58 \\
\hline \multirow[t]{3}{*}{$\mathrm{S} 2$} & 71,50 & 70,75 & 63,25 & 1,64 & 1,66 & 1,61 \\
\hline & \multicolumn{3}{|c|}{ Matéria seca da parte aérea $(\mathrm{g})$} & \multicolumn{3}{|c|}{ Matéria seca de raízes $(\mathrm{g})$} \\
\hline & Testemunha & G. clarum & A. Scrobiculata & Testemunha & G. clarum & A. Scrobiculata \\
\hline S1 & $0,93 \mathrm{bB}$ & $0,98 \mathrm{abB}$ & $1,25 \mathrm{aA}$ & $1,07 \mathrm{bB}$ & $1,30 \mathrm{abA}$ & $1,61 \mathrm{aA}$ \\
\hline S2 & $1,51 \mathrm{aA}$ & $1,38 \mathrm{abA}$ & $1,30 \mathrm{bA}$ & $1,43 \mathrm{aA}$ & $1,30 \mathrm{aA}$ & $1,17 \mathrm{bB}$ \\
\hline
\end{tabular}

Médias seguidas de mesma letra, minúscula na linha e maiúscula na coluna, não diferem entre si, ao nível de 5\%, pelo teste de Duncan. S1: solo + areia, 1:1, v:v. S2: solo + areia + resíduo decomposto de casca de acácia-negra, 2:2:1, v:v:v.

O melhor desenvolvimento vegetativo das plantas cultivadas no $\mathrm{S} 2$ pode ser atribuído às condições físicas e químicas do substrato (Schäfer, 2004; Fochesato, 2005). Em substratos pobres nutricionalmente, os FMAs são importantes, pois aceleram o desenvolvimento das plantas, uma vez que as hifas micorrízicas externas às raízes funcionam como extensão do sistema radicular, aumentando sua capacidade em explorar maior volume de solo, possibilitando a absorção maior de nutrientes do mesmo (Rosand \& Dias, 1985), situação verificada nas plantas cultivadas no S1. Por sua vez, a adição de resíduo decomposto de casca de acácia no substrato anulou a ação dos FMAs, inclusive tornando-os prejudiciais, no caso de $A$. scrobiculata, cultivada no S2. Esse comportamento pode ser explicado pela nutrição do substrato, onde a planta cultivada em substrato rico em nutrientes pode ser independente dos FMAs para absorvê-los. Nessas condições, os FMAs podem ser prejudiciais, pois, além de não favorecerem a absorção nutricional, podem consumir reservas das plantas, o que é visualizado na Figura 1

Verifica-se que, mesmo sendo prejudicial às plantas cultivadas no S2, a colonização com FMA foi elevada (entre 80 e $95 \%$ das radicelas), o que significa que a pouca eficiência da simbiose planta-FMA não necessariamente passa pela baixa colonização radicular. Fica evidente, nesse caso, que com um nível semelhante de colonização radicular as espécies de FMA foram ineficazes nas plantas cultivadas no S2, atuando como parasitas, enquanto, no S1, mostraram um comportamento positivo.

A resposta obtida com os FMAs foi altamente variável em função do substrato estudado. Esse fato indica que as características do substrato, especialmente teor de matéria orgânica, porosidade total, espaço de aeração e disponibilidade de água, afetam a eficácia dos FMA, em conformidade com Souza et al. (2003).

\section{CONCLUSÕES}

1. A adição de resíduo decomposto de casca de acácia melhorou as características químicas e físicas de um substrato constituído de solo + areia, permitindo maior desenvolvimento vegetativo e conteúdo de reservas às plantas de Flying dragon.

2. A ação dos FMAs testados sobre o desenvolvimento vegetativo e substâncias de reserva de Flying dragon é dependente do substrato e da espécie de FMA, recomendando-se o uso de $A$. scrobiculata para Flying dragon somente se usado substrato solo + areia.

\section{REFERÊNCIAS}

DE BOODT, M.; VERDONCK, O. The physical properties of the substrates in horticulture. Acta Horticulturae, Wageningen, v.1, n.23, p.37-44, 1972.

EMBRAPA. Sistema Brasileiro de Classificação de Solos. Brasília: EMBRAPA, 1999.412p.
FOCHESATO, M. L. Substratos e porta-enxertos na produção de mudas cítricas em ambiente protegido. 2005. 91f. Dissertação (Mestrado em Agronomia - Fitotecnia) - Faculdade de Agronomia, Universidade Federal do Rio Grande do Sul, Porto Alegre, 2005.

ISHII, T.; KADOYA, K. Effects of charcoal as a soil conditioner on citrus growth and vesicular-arbuscular mycorrhizal development. Journal of the Japanese Society for Horticultural Science, Tokyo, v.63, n.3, p.529-535, 1994.

KÄMPF, A.N. Produção comercial de plantas ornamentais. Guaíba: Agropecuária, 2000. 254p.

KOLLER, O.C. Citricultura: laranja, limão e tangerina. Porto Alegre: Rígel, 1994. 446p.

OLIVEIRA, J.B. Solos para citros. In: RODRIGUES, O.; VIEGAS, F.; POMPEU JÚNIOR, J. et al. Citricultura brasileira. Campinas: Fundação Cargill, 1991. v.1, p.196-227.

PENNINGSFELD, F. Kulturusubstrate fur den gartenbau, besonders in Deutschland: ein kritischer Jberblick. Plant and Soil, The Hague, v.75, n 3, p. 269-281, 1983.

PROGRAMAESTADUAL DE CITRICULTURA. Montenegro. CECRGS, 1989.48p.

ROSAND, P.C.; DIAS, R. Associações micorrízicas e a nutrição mineral das plantas. In: REUNIÃO BRASILEIRA SOBRE MICORRIZAS, 1., 1985, Lavras. Anais... Lavras: FAEPE, 1985. p.33-59.

SCHÄFER, G. Produção de porta-enxertos cítricos em recipientes e ambiente protegido no Rio Grande do Sul. 2004. 129f. Tese (Doutorado em Agronomia - Fitotecnia)- Universidade Federal do Rio Grande do Sul, Porto Alegre, 2004.

SCHÄFER, G.; DORNELLES. A.L.C. Produção de mudas cítricas no Rio Grande do Sul - Diagnóstico da região produtora. Ciência Rural, Santa Maria, v. 30, n. 4, p. 587-592, 2000.

SCHMITZ, J.A.K.; SOUZA, P.V.D.; KÄMPF, A.N. Propriedades químicas e físicas de substratos de origem mineral e orgânica para cultivo de mudas em recipientes. Ciência Rural, Santa Maria, v. 32, n. 6, p. 937-944, 2002.

SOUZA, P. V. D. de Efeito de Concentração de etefon e pressões de pulverização foliar no raleio de frutinhos em tangerineiras (Citrus deliciosa Tenore) cv. Montenegrina. 1990. 139f. Dissertação (Mestrado em Agronomia - Fitotecnia). Universidade Federal do Rio Grande do Sul, Porto Alegre, 1990.

SOUZA, P.V.D.; CARNIEL, E.; SCHMITZ, J.A.K.; SILVEIRA, S.V. Substratos e fungos micorrízicos arbusculares no desenvolvimento vegetativo de Citrange Troyer. Agropecuária Catarinense, Florianópolis, v. 16, n.3, p. 84-88, 2003.

VERDONCK, O.; VLEESCHAUMER, D.; DE BOODT, M. The influence of the substrate to plant growth. Acta Horticulturae, Wageningen, v. 1, n. 150, p.467-473, 1981. 